\title{
DESTILAÇÃO COM MEMBRANAS APLICADA PARA CONCENTRAÇÃO DE SOLUÇÕES AQUOSAS DE CLORETO DE SÓDIO: MODELAGEM MATTEMÁTICA
}

\author{
Y. N. NARIYOSHI ${ }^{1}$, C. E. PANTOJA ${ }^{1}$, M. M. SECKLER ${ }^{1}$ \\ ${ }^{1}$ Universidade de São Paulo, Departamento de Engenharia Química \\ E-mail: yuri.nn@usp.br
}

\begin{abstract}
RESUMO - Um modelo matemático foi desenvolvido para predição do fluxo transmembrana de vapor de água em processos de destilação com membranas (DM). O modelo se ajustou favoravelmente a dados experimentais com soluções aquosas concentradas de cloreto de sódio $(\mathrm{NaCl})$. Devido à elevada concentração salina, um método termodinâmico robusto foi empregado para a correta predição da pressão de vapor da água. Também foram considerados efeitos de polarização de temperaturas e de concentração. $\mathrm{O}$ modelo mostrou ser uma ferramenta útil para prever o comportamento da DM em altas concentrações salinas, que é necessário para o desenvolvimento de processos de cristalização assistida por destilação com membranas (CDM).
\end{abstract}

\section{INTRODUÇÃO}

Em unidades de processamento químico e petroquímico é cada vez mais comum a presença de sistemas de tratamento terciário de efluentes aquosos visando a maior recuperação de água possível. No caso de complexos petroquímicos, a reutilização de água requer a remoção de matéria orgânica recalcitrante e de sais dissolvidos. Para este último, processos de separação por membranas, tais como osmose reversa e eletrodiálise têm sido preferencialmente aplicados (Fornari e Godoi, 2012). Essas tecnologias geralmente permitem a produção de água de elevada pureza, contudo geram como rejeito uma corrente aquosa concentrada de sais (retentato), que pode representar de 20 a 30\% do volume da corrente de alimentação (Baker, 2004). Esses retentatos são frequentemente dispostos em lagoas de evaporação, injetados em poços profundos ou descartados em águas costeiras (Kim, 2011). Devido às crescentes restrições ambientais, num futuro próximo será necessário tratar estes concentrados salinos de forma a se recuperar totalmente a água e dispor os sais dissolvidos como material particulado sólido, um problema de separação que requer a concentração adicional do retentato e a cristalização dos sais. Estas duas operações podem ser realizadas sequencialmente ou combinadas num só processo de CDM, o qual permite o emprego de fontes residuais de energia de baixa entalpia.

O processo de CDM é baseado na evaporação sem ebulição da água através dos poros cheios de ar de uma membrana hidrofóbica. O vapor escoa da corrente de retentato (solução salina) para a corrente de destilado (água pura), correntes estas posicionadas em lados opostos da membrana. O retentato salino, que se torna supersaturado como consequência da evaporação, escoa para um cristalizador onde essa supersaturação é aliviada (Curcio, Criscuoli e Drioli, 2001). 
Tanto o desenvolvimento de processos DM quanto CDM requerem que se conheçam os mecanismos que controlam o transporte de vapor desde o retentato até o destilado, bem como o valor do fluxo transmembrana. Nesse contexto, propõe-se neste trabalho desenvolver um modelo matemático do processo de DM para predição com acurácia do fluxo transmembrana, bem como comparar o modelo com experimentos em escala de bancada para o fluxo de vapor de água a partir de soluções aquosas de $\mathrm{NaCl}$. Este sal foi escolhido por possuir solubilidade elevada, além de ser um dos constituintes majoritários em efluentes terciários oriundos de grande parte das indústrias de processamento químico e petroquímico.

\section{DESENVOLVIMENTO DO MODELO}

O fluxo transmembrana de vapor de água estabelecido através dos microporos de uma membrana hidrofóbica na configuração de destilação com membranas por contato direto (DMCD), configuração mais simples e recomendada para tratamento de soluções salinas (Figura 1), pode ser expresso pela Equação 1.

$$
F_{M}=K_{M} \cdot\left(p_{1}-p_{0}\right)
$$

Nesta equação o fluxo é proporcional à diferença de pressão de vapor da água entre as interfaces líquido-vapor em lados opostos dos poros da membrana (força motriz do processo).

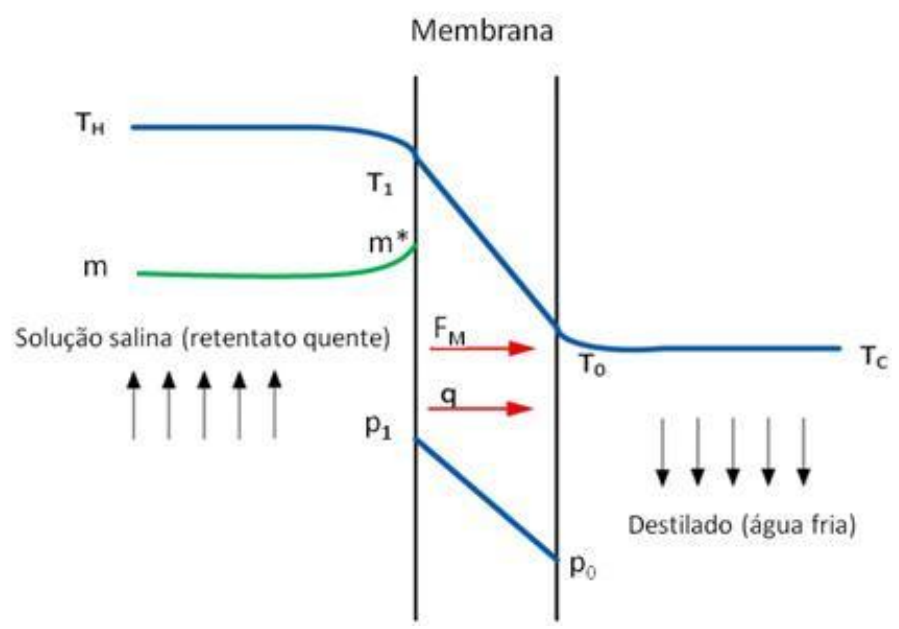

Figura 1 - Perfis de temperatura $(T)$, de concentração molal $(m)$ e de pressão de vapor $(p)$ em processo de DMCD, em que $F_{M}$ indica fluxo de massa e $q$ fluxo de calor. Os subscritos 1 e 0 indicam respectivamente a interface quente e fria. $T_{H}$ e $T_{C}$ são as temperaturas nos seios dos líquidos em circulação, e $m$ * é a concentração molal na interface do retentato.

No processo DMCD, os poros da membrana são preenchidos com ar atmosférico, e, portanto, a resistência à difusão ordinária das moléculas de vapor de água através da camada de ar estagnado $\left(K_{D}\right)$ tem papel importante na descrição do coeficiente global de transferência de massa $\left(K_{M}\right)$. Além disso, considerando que o raio dos poros $(r)$ das membranas tipicamente utilizadas em DMCD situam-se entre 0.10 e $0.45 \mu \mathrm{m}$, e que por outro lado o caminho livre médio de uma molécula de água a $60^{\circ} \mathrm{C}$ é da ordem de $0.30 \mu \mathrm{m}$, também deve ser considerada a resistência difusiva de Knudsen $\left(K_{K}\right)$ (Schofield, Fane e Fell, 1987). Conforme descrito por Alkhudhiri, Darwish e Hilal (2012), estas resistências podem ser associadas em série, em uma 
analogia com circuitos elétricos, para determinação do coeficiente global $K_{M}$. As correlações são apresentadas na Tabela 1. Estas requerem informações características da membrana como porosidade $(\varepsilon)$, tortuosidade $(\chi)$, espessura $(\delta)$ e raio de poro $(r)$. Também requerem dados físico-químicos como difusividade do vapor de água no ar $\left(D_{w}\right)$, massa molar da água $\left(M_{w}\right)$, constante dos gases ideais $(R)$, média logarítmica da fração molar de ar $\left(Y_{l n}\right)$ e média aritmética da temperatura $\left(T_{m}\right)$ dentro dos poros.

Tabela 1 - Correlações de transferência de massa envolvidas no processo DMCD

\begin{tabular}{|c|c|c|}
\hline $\begin{array}{c}\text { Coeficiente global de } \\
\text { transferência de massa }\end{array}$ & $\begin{array}{c}\text { Resistência difusiva } \\
\text { ordinária }\end{array}$ & $\begin{array}{c}\text { Resistência difusiva de } \\
\text { Knudsen }\end{array}$ \\
\hline$K_{M}=\left(\frac{Y_{l n}}{K_{D}}+\frac{1}{K_{K}}\right)^{-1}$ & $K_{D}=\frac{1}{Y_{l n}}\left(\frac{D_{w} \varepsilon}{\chi \delta}\right)\left(\frac{M_{w}}{R T_{m}}\right)$ & $K_{K}=1.064\left(\frac{r \varepsilon}{\chi \delta}\right)\left(\frac{M_{w}}{R T_{m}}\right)^{0.5}$ \\
\hline
\end{tabular}

A pressão de vapor em cada interface da membrana em equilíbrio líquido-vapor (ELV) pode ser estimada pela equação de Antoine (Tabela 2). Esta depende da temperatura em cada interface $\left(T_{1}\right.$ e $\left.T_{0}\right)$ e dos parâmetros $A, B$ e $C$ específicos para a água. No lado da solução salina, as espécies iônicas dissolvidas provocam abaixamento da pressão de vapor quando comparada à pressão de vapor da água pura na mesma temperatura, sendo tal abaixamento proporcional à atividade da água $\left(a_{w}\right)$.

Tabela 2 - Equação de Antoine aplicada em cada interface da membrana em ELV

\begin{tabular}{|c|c|}
\hline $\begin{array}{c}\text { Pressão de vapor na interface } \\
\text { solução salina-vapor }\end{array}$ & $\begin{array}{c}\text { Pressão de vapor na interface } \\
\text { água-vapor }\end{array}$ \\
$p_{1}=a_{W} \cdot 10^{A-\frac{B}{C+T_{1}}}$ & $p_{0}=10^{A-\frac{B}{C+T_{0}}}$ \\
\hline
\end{tabular}

As temperaturas interfaciais $T_{1}$ e $T_{0}$ diferem respectivamente das temperaturas $T_{H}$ e $T_{C}$ nos seios dos líquidos que escoam por ambos os lados da membrana, devido às camadas limite térmicas que se estabelecem nas interfaces. A título de simplificação, serão desconsideradas as variações de temperatura longitudinais. Será considerada apenas uma camada limite térmica em cada lado da membrana. As temperaturas nessas camadas limite térmicas estão diretamente relacionadas com o fluxo de calor radial, ou seja,

$$
q=h_{1} \cdot\left(T_{H}-T_{1}\right) \quad \text { e } \quad q=h_{0} \cdot\left(T_{0}-T_{C}\right)
$$

Onde $h_{1}$ e $h_{0}$ são os coeficientes convectivos de troca térmica do lado do retentato e destilado, respectivamente. Um balanço de energia em regime permanente impõe que o fluxo de calor radial $q$ seja igual à variação de entalpia do fluido ao atravessar o módulo de membranas, isto é:

$$
q=F_{H} \cdot c_{p, H} \cdot \Delta T_{H} \quad \text { e } \quad q=F_{C} \cdot c_{p, C} \cdot \Delta T_{C}
$$

Onde $F_{H}$ e $F_{C}$ são as respectivas vazões mássicas do retentato e do destilado, $\Delta T_{H}$ e $\Delta T_{C}$ são as respectivas variações de temperaturas das correntes de retentato e destilado e $c_{p, H}$ e $c_{p, C}$ são os respectivos calores específicos. As equações (2) e (3) são empregadas para se determinar as temperaturas interfaciais $T_{1}$ e $T_{0}$. Para isso, os coeficientes convectivos $h_{1}$ e $h_{0}$ 
são calculados a partir do número de Nusselt característico para cada lado da membrana, que depende do regime e da geometria de escoamento. Neste modelo será desprezada a contribuição geométrica. Existem várias correlações empíricas disponíveis para esta finalidade, sendo que para o presente são adotadas as sugeridas por Phattaranawik et al., (2003), conforme mostra a Tabela 3. Estas são calculadas a partir do comprimento característico $(L)$ das membranas, da condutividade térmica $(k)$ dos fluídos, do diâmetro equivalente $\left(D_{e}\right)$ e dos números de Reynolds $(R e)$ e de Prandt $(P r)$.

Tabela 3 - Correlações empíricas adotadas para cálculo do número de Nusselt

\begin{tabular}{|c|c|}
\hline Regime laminar & Regime turbulento \\
\hline$N u=\frac{h \cdot L}{k}=4.36+\frac{0.036 \cdot \operatorname{Re} \cdot \operatorname{Pr} \cdot\left(d_{e} / L\right)}{1+0.0011 \cdot\left[\operatorname{Re} \cdot \operatorname{Pr} \cdot\left(d_{e} / L\right)\right]^{0.8}}$ & $N u=\frac{h \cdot L}{k}=0.023 \cdot\left(1+\frac{6 d_{e}}{L}\right) \cdot \operatorname{Re}^{0.8} \cdot \operatorname{Pr}^{1 / 3}$ \\
\hline
\end{tabular}

As resistências convectivas à transferência de calor nas duas camadas limite térmicas são responsáveis pelo fenômeno de polarização de temperaturas, caracterizado por um coeficiente de polarização de temperaturas $(C P T)$. Este relaciona a diferença de temperaturas no seio dos líquidos em escoamento com a diferença efetiva de temperaturas nas interfaces da membrana em ELV (Tun et al., 2005). De forma análoga, a vaporização da água, que ocorre na interface da membrana do lado do retentato, provoca uma variação local de concentração do soluto em relação à sua concentração no seio da solução. Esta variação dá origem à polarização de concentração, que pode ser quantificada pelo chamado coeficiente de polarização de concentração (CPC) (Ji et al., 2010). Este relaciona a concentração molal de soluto na interface $\left(m^{*}\right)$ com a concentração no seio líquido $(m)$ em função da densidade da solução $\left(\rho_{H}\right)$, do fluxo transmembrana $\left(F_{M}\right)$ e do coeficiente de transporte de massa do soluto $\left(K_{L}\right)$. Ambos coeficientes $C P T$ e $C P C$ são apresentados pelas Equações (4) a seguir:

$$
C P T=\frac{T_{1}-T_{0}}{T_{H}-T_{C}} \quad \text { e } \quad C P C=\frac{m^{*}}{m}=e^{\left(\frac{F_{M}}{\rho_{H} \cdot K_{L}}\right)}
$$

O coeficiente de transporte de massa do soluto $K_{L}$ da Equação (4) pode ser calculado por correlações semi-empíricas como as propostas por Dittus-Boelter para regime turbulento e por Levesque para regime laminar (Yun et al., 2006), conforme mostra a Tabela 4. Para este cálculo são necessários dados como velocidade de escoamento $(v)$, comprimento característico $(L)$, diâmetro equivalente $\left(d_{e}\right)$, difusividade do $\mathrm{NaCl}\left(D_{\mathrm{NaCl}}\right)$ e números de Reynolds $(R e)$ e Schmidt $(S c)$.

Tabela 4 - Correlações semi-empíricas adotadas para cálculo do $K_{L}$

\begin{tabular}{|c|c|}
\hline Regime laminar & Regime turbulento \\
\hline$K_{L}=1.62 \cdot\left(\frac{d_{e} \cdot V}{L}\right)^{1 / 3} \cdot D_{\mathrm{NaCl}}^{2 / 3}$ & $K_{L}=0.023 \cdot \mathrm{Re}^{0.8} \cdot S c^{0.33} \cdot \frac{D_{\mathrm{NaCl}}}{d_{e}}$ \\
\hline
\end{tabular}

A predição da atividade da água $\left(a_{w}\right)$ é fundamental para a correta estimativa da pressão de vapor no lado do retentato, já que esta variável faz parte da força motriz para o fluxo de vapor. O presente modelo considera um método termodinâmico rigoroso para o cálculo da atividade da água em sistemas aquosos de eletrólitos fortes: o método de Pitzer (Zemaitis, 
1986). Este método possui como entradas a composição e temperatura da solução salina, fornecendo como resultado o coeficiente osmótico da água $(\phi)$, que por sua vez tem relação direta com a atividade da água $a_{w}$. Os detalhes do método não serão, entretanto, aqui apresentados.

Nos módulos do tipo fibras ocas comumente empregados em DMCD, a concentração da solução salina aumenta ao longo do mesmo à medida que água é evaporada. Da mesma forma, devido ao contato direto dos fluidos com as membranas em diferentes temperaturas, a temperatura do retentato tende a diminuir e a temperatura do destilado tende a aumentar ao longo do módulo. Por conta da alta vazão de recirculação, tais variações serão desprezadas. Ou seja, serão consideradas vazões constantes, médias logarítmicas de diferença de temperaturas (MLDT) no seio líquido de ambos os lados das membranas e concentração salina média do retentato ao longo do módulo. Relações adicionais para as propriedades termofísicas dos fluidos são parte integrante do modelo, não sendo, entretanto, aqui apresentadas. O modelo resultante é, portanto, composto por um conjunto de equações algébricas que devem ser resolvidas simultaneamente.

\section{VALIDAÇÃO DO MODELO}

A título de validação do modelo foi testada sua aderência a dados experimentais em uma unidade semi-contínua em escala de bancada. Esta operou com um módulo comercial de membranas de microfiltração (Microdyn ${ }^{\circledR}$, MD020CP2N), contendo 40 membranas hidrofóbicas de polipropileno do tipo fibras ocas. De acordo com o fabricante, as membranas possuem porosidade de $70 \%$, tamanho nominal de poro de $0.2 \mu \mathrm{m}$, espessura de $450 \mu \mathrm{m}$, diâmetro externo de $1.8 \mathrm{~mm}$ e área disponível de $0.1 \mathrm{~m}^{2}$. As condições experimentais foram variadas conforme apresenta a Tabela 5. Os ensaios foram conduzidos durante 30 minutos após alcançado o regime permanente.

Tabela 5 - Condições experimentais dos ensaios de DMCD

\begin{tabular}{|c|c|c|}
\hline Alimentação & Água destilada & Solução aquosa NaCl \\
\hline Concentração inicial de NaCl & 0 & $10,12,30,33$ e $36 \mathrm{~kg} / 100 \mathrm{~kg}$ água \\
\hline Alocação nas membranas & Lado externo & Lado interno \\
\hline Recirculação retentato & 50,100 e $200 \mathrm{~L} / \mathrm{h}$ & $200 \mathrm{~L} / \mathrm{h}$ \\
\hline Recirculação destilado & 50,100 e $200 \mathrm{~L} / \mathrm{h}$ & 50 e $190 \mathrm{~L} / \mathrm{h}$ \\
\hline Temperatura entrada retentato & $30,40,45$ e $50{ }^{\circ} \mathrm{C}$ & 35 e $40{ }^{\circ} \mathrm{C}$ \\
\hline Temperatura entrada destilado & $15,25,30$ e $35^{\circ} \mathrm{C}$ & 20 e $28^{\circ} \mathrm{C}$ \\
\hline
\end{tabular}

\section{RESULTADOS E DISCUSSÃO}

Os gráficos apresentados pelas Figuras 2 e 3 a seguir reportam os dados de fluxo transmembrana calculados pelo modelo e medidos experimentalmente em função da diferença de pressão de vapor calculada nas interfaces dos poros da membrana em ELV para as condições experimentais previamente apresentadas. É possível observar que o modelo se ajustou bem aos valores experimentais, tanto para ensaios com água pura (Figura 2) quanto para os ensaios com soluções salinas diluídas e concentradas de $\mathrm{NaCl}$ (Figura 3), para amplas faixas de temperaturas do lado do destilado e do retentato, para diferentes taxas de 
recirculação e em diferentes alocações da alimentação nas membranas. Nota-se também que o modelo superestima levemente o fluxo transmembrana (em torno de 10\%). Entretanto, ambas retas modeladas possuem as mesmas inclinações das respectivas retas experimentais, o que valida o coeficiente global de transferência de massa da membrana $\left(K_{M}\right)$ adotado. O mecanismo dominante no transporte de massa foi a difusão ordinária, uma vez o termo da difusão de Knudsen $\left(1 / K_{K}\right)$ apresentou valores em torno de $3.93 \times 10^{2} \mathrm{~s} / \mathrm{m}$ enquanto o termo da difusão ordinária $\left(Y_{l n} / K_{D}\right)$ em torno de $5.12 \times 10^{6} \mathrm{~s} / \mathrm{m}$. Para os ensaios com água destilada, o menor fluxo transmembrana obtido foi de 0.7 e o maior de $3.2 \mathrm{~kg} \cdot \mathrm{m}^{-2} \cdot \mathrm{h}^{-1}$, com a diferença de pressão de vapor variando de 1.3 a $4.4 \mathrm{kPa}$. Em relação aos ensaios com soluções diluídas e concentradas de $\mathrm{NaCl}$, o menor fluxo transmembrana obtido foi de 0.6 e o maior de $2.0 \mathrm{~kg} . \mathrm{m}^{-}$ ${ }^{2} \cdot \mathrm{h}^{-1}$, com a diferença de pressão de vapor de 1.0 a $2.9 \mathrm{kPa}$.

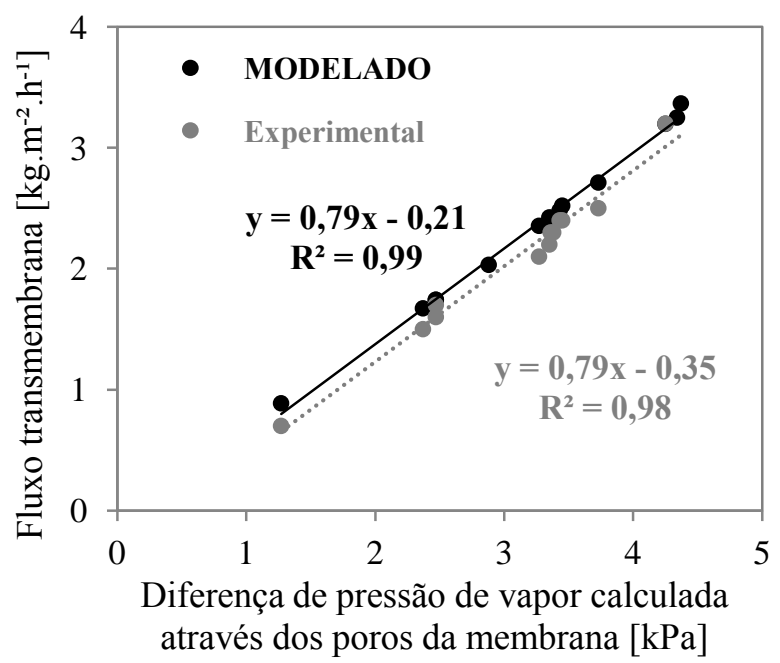

Figura 1 - Dados de fluxo transmembrana em função da diferença de pressão de vapor para o processo DMCD alimentado com água pura.

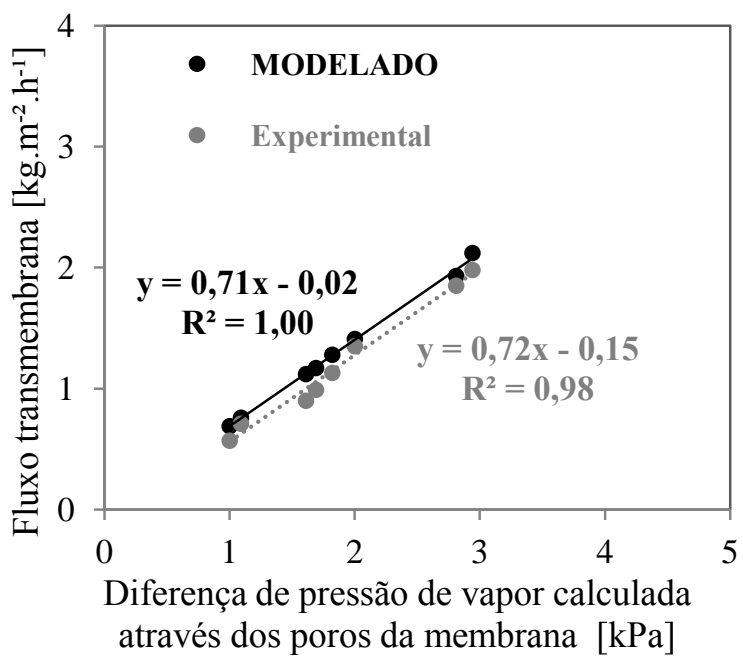

Figura 2 - Dados de fluxo transmembrana em função da diferença de pressão de vapor para o processo DMCD alimentado com soluções aquosas de $\mathrm{NaCl}$.

O valor de $K_{M}$ para soluções aquosas de $\mathrm{NaCl}$ é ligeiramente menor ao valor para água pura $\left(0.72<0.79\right.$ h.m $\left.\mathrm{m}^{-1}\right)$. Esta diferença ocorre porque o $K_{M}$ é inversamente proporcional ao termo dominante do transporte de massa $\left(Y_{l n} / K_{D}\right)$, uma vez que a presença do sal aumenta a $Y_{l n}$, ocorre o consequente abaixamento do $K_{M}$. A Tabela 6 a seguir mostra que tanto a concentração quanto a temperatura da solução impactam diretamente o valor de $Y_{l n}$. Quanto menor a temperatura da solução e maior a concentração de sal, maior será o $Y_{l n}$ e menor será o $K_{M}$

Tabela 6 - Dados da corrente de solução salina atravessando o módulo de membranas

\begin{tabular}{|c|c|c|}
\hline $\begin{array}{c}\text { Concentração } \\
{[\mathrm{kg} \mathrm{NaCl} / \mathrm{kg} \text { água }]}\end{array}$ & $\begin{array}{c}\text { Temperatura } \\
\text { média }\left[{ }^{\circ} \mathrm{C}\right]\end{array}$ & $\begin{array}{c}Y_{l n} \\
{[-]}\end{array}$ \\
\hline 0.10 & 34 & 0.960 \\
\hline 0.11 & 44 & 0.935 \\
\hline 0.30 & 34 & 0.964 \\
\hline 0.35 & 44 & 0.943 \\
\hline
\end{tabular}


O CPT no módulo de membranas variou pouco de 0.60 a 0.70 , indicando que o gradiente de temperaturas nas interfaces da membrana são 40 e $30 \%$ menores que os respectivos gradientes de temperaturas nos seios líquidos. Em relação ao $C P C$, este variou de 1.02 a 1.08, indicando que a concentração na interface quente da membrana chegou a ser $8 \%$ maior que concentração no seio líquido da solução. Em relação ao impacto dessas polarizações no fluxo, a Tabela 7 apresenta a contribuição individual de cada fenômeno. Observa-se que o fluxo real medido sofre redução total de 42 a 66\% com base no seu valor ausente de polarizações (fluxo ideal). A polarização de temperaturas é mais pronunciada em soluções diluídas, representando em torno de $32 \%$ da redução total do fluxo ideal. No que diz respeito à polarização de concentrações, esta se torna dominante quando se trata de soluções concentradas, chegando a representar em torno de $40 \%$ da redução total do fluxo ideal.

Tabela 7 - Efeito dos fenômenos de polarização no fluxo transmembrana

\begin{tabular}{|c|c|c|c|}
\hline $\begin{array}{c}\text { Concentração inicial } \\
\text { de } \mathrm{NaCl}[\mathrm{kg} / \mathrm{kg} \text { água }\end{array}$ & $\begin{array}{c}\text { Redução total do } \\
\text { fluxo ideal [\%] }\end{array}$ & $\begin{array}{c}\text { Redução do fluxo } \\
\text { devido ao CPT [\%] }\end{array}$ & $\begin{array}{c}\text { Redução do fluxo } \\
\text { devido ao CPC [\%] }\end{array}$ \\
\hline 0.10 & -42.5 & -31.4 & -11.1 \\
\hline 0.11 & -47.9 & -36.5 & -11.4 \\
\hline 0.12 & -42.7 & -28.3 & -14.4 \\
\hline 0.30 & -65.3 & -27.2 & -38.1 \\
\hline 0.31 & -64.8 & -25.4 & -39.4 \\
\hline 0.34 & -63.9 & -22.9 & -41.0 \\
\hline 0.35 & -66.2 & -22.7 & -43.5 \\
\hline
\end{tabular}

\section{CONCLUSÃO}

Um modelo matemático foi desenvolvido com objetivo de predizer o fluxo transmembrana de vapor de água em processos de destilação por membranas. A força motriz é a diferença de pressão de vapor através dos poros, a qual é calculada por abordagem termodinâmica rigorosa realizada pelo método de Pitzer. O modelo foi comparado a dados experimentais para soluções $\mathrm{NaCl}-\mathrm{H}_{2} \mathrm{O}$ com concentrações até a saturação. O mecanismo dominante de transporte de massa na membrana foi a difusão ordinária, afetada de forma mais pronunciada pela polarização de temperaturas em soluções diluídas e pela polarização de concentração em soluções concentradas. Apesar das simplificações realizadas, o modelo superestimou apenas levemente os fluxos (em torno de 10\%), se mostrando uma ferramenta útil para projeto de processos de DM envolvendo cristalização (CDM).

\section{REFERÊNCIAS}

ALKHUDHIRI, A.; DARWISH, N.; HILAL, N. Membrane distillation: A comprehensive review. Desalination, v. 287, p. 2-18, FEB 152012 2012. ISSN 0011-9164.

BAKER, R. W. Membrane technology and applications Second edition. West Sussex: John Wiley \& Sons, 2004. 535 ISBN 0470854456.

CURCIO, E.; CRISCUOLI, A.; DRIOLI, E. Membrane crystallizers. Industrial \& Engineering Chemistry Research, v. 40, n. 12, p. 2679-2684, Jun 13 2001. ISSN 08885885. Disponível em: $<<$ Go to ISI $>$ ://WOS:000169279200015 $>$.

FORNARI, M.; GODOI, L. Cresce reuso de água no Brasil. Saneamento ambiental, n. 162, p. $12-15,2012$. 
JI, X. et al. Membrane distillation-crystallization of seawater reverse osmosis brines. Separation and Purification Technology, v. 71, n. 1, p. 76-82, Jan 2010. ISSN 13835866. Disponível em: $<<$ Go to ISI $>$ ://WOS:000274495200011 $>$.

KIM, D. A review of desalting process techniques and economic analysis of the recovery of salts from retentates. Desalination, v. 270, n. 1-3, p. 1-8, APR 12011 2011. ISSN 00119164.

PHATTARANAWIK, J.; JIRARATANANON, R.; FANE, A. Heat transport and membrane distillation coefficients in direct contact membrane distillation. Journal of Membrane Science, v. 212, n. 1-2, p. 177-193, FEB 152003 2003. ISSN 0376-7388.

SCHOFIELD, R.; FANE, A.; FELL, C. Heat and mass transfer in membrane distillation. Journal of Membrane Science, v. 33, n. 3, p. 299-313, OCT 1987 1987. ISSN 03767388.

TUN, C. et al. Membrane distillation crystallization of concentrated salts - flux and crystal formation. Journal of Membrane Science, v. 257, n. 1-2, p. 144-155, JUL 1520052005. ISSN 0376-7388.

YUN, Y. et al. Direct contact membrane distillation mechanism for high concentration $\mathrm{NaCl}$ solutions. Desalination, v. 188, n. 1-3, p. 251-262, FEB 82006 2006. ISSN 0011-9164.

ZEMAITIS, J. R. Handbook of Aqueous Electrolyte Thermodynamics. New York: AIChE, 1986. 852 . 